\title{
Surface-Enhanced Raman Scattering of the Complexes of Silver with Adenine and dAMP
}

\author{
C. Otto,* F. P. Hoeben and J. Greve \\ University of Twente, Faculty of Applied Physics, Biophysical Technology Group, P.O. Box 217, 7500 AE Enschede, The \\ Netherlands
}

\begin{abstract}
The behaviour of adenine and $2^{\prime}$-deoxyadenosine-5'-monophosphate (dAMP) at positive surface potentials of a silver working electrode was investigated using surface-enhanced Raman scattering (SERS). The use of positive potentials in the presence of adenine or dAMP leads to a rapid accumulation of an intense spectrum. It is proposed that complexes of adenine (dAMP) with silver generate the observed spectra. Adenine and dAMP can be distinguished spectroscopically due to various different complexes that can be formed for adenine and not for dAMP. The SERS spectra of the silver-adenine complexes show broad, unresolved bands between 1200 and $1500 \mathrm{~cm}^{-1}$, while the spectra of complexes of silver with dAMP show clearly resolved bands. The formation of $\mathrm{Ag}^{+}-\mathrm{adenine}^{-}$ complexes gives rise to a decrease in $\mathrm{pH}$ and must therefore be associated with deprotonation of the adenine ring system. The SERS spectra of adenine can be obtained in solutions with a pH of 3.0, 7.5 and 11.0 whereas the SERS spectra of dAMP can only be obtained at a $\mathrm{pH}$ of 7.5 and 11.0. The time dependence of the SERS spectra of dAMP suggests that at least two different complexes of silver with dAMP are involved. The observed spectra are completely different from the 'classical' SERS spectrum of adenine and dAMP. This spectrum is characterized by an intense band at $729 \mathrm{~cm}^{-1}$ (at $-700 \mathrm{mV}$ vs. SCE). At open loop potential, however, the spectra of the Ag-dAMP complex change slowly to the 'classical' SERS spectrum of dAMP.
\end{abstract}

\section{INTRODUCTION}

The high gain in Raman scattering intensity and the virtual absence of fluorescence under surface-enhanced Raman scattering (SERS) conditions has led to many applications of SERS in the field of biophysics. This topic has recently been reviewed. ${ }^{1}$

The application of SERS is a relatively easy method for obtaining vibrational information on monolayers of material. As many biophysical processes take place at interfaces, the application of SERS to these processes may provide valuable information on the changes in the chemical constitution at the interface. However, as has recently been pointed out by Nabiev et al., ${ }^{2}$ a knowledge of the actual structure and activity of biological macromolecules at the SERS interface is a matter of the greatest importance.

Two systems providing a solid/liquid interface have been used widely for the study of biological molecules: a three-electrode electrochemical cell and suspensions of colloids. These systems differ in the way silver particles with a suitable curvature are generated. A typical particle radius of $10-50 \mathrm{~nm}$ gives rise to high local field strengths at the surface and therefore high-intensity SERS spectra. ${ }^{3}$

Both silver colloids and electrochemically prepared surfaces have been used to obtain SERS spectra of native and denatured $\mathrm{DNA}^{4-8}$ of the compounds of which DNA is composed, i.e. the nucleotides and nucleosides ${ }^{4,6,9,10}$ and also of the free base molecules: adenine (A), thymine (T), guanine (G), cytosine (C) and uracil $(\mathrm{U}) .^{4-7,9-14}$

\footnotetext{
* Author to whom correspondence should be addressed
}

The interaction of metals with nucleic acids has been reviewed. ${ }^{15}$ A classification of metals according to the degree of electronegativity has been proposed. ${ }^{16}$ Silver ions belong to the 'class b' metals, i.e. metals which act as strong Lewis acids. In adenine the sites which are most vulnerable for silver-ion attack are the $\mathrm{N}-1, \mathrm{~N}-7$ and $\mathrm{N}-9$ atoms, while specific interactions of $\mathrm{Ag}^{+}$with dAMP in nucleic acids may be directed to the N-1 and $\mathrm{N}-7$ sites.

In an electrochemical cell, the surface potential can be controlled. The position of vibrational bands changes slightly as a function of the surface potential. This has been used to infer the potential at the surface of a silver colloidal particle. In this way it has been shown that the surface potential is ca. $+120 \mathrm{mV} .{ }^{17}$ The chemical constitution of the surface in an electrochemical three-electrode system can be manipulated and investigated using the vibrational information in the SERS spectrum. On the other hand, the signal intensity in colloid-related SERS experiments seems crucially dependent on the morphology of the sol, ${ }^{10,18,19}$ that is, a certain amount of aggregation is necessary to produce strong SERS signals. This aggregation can be induced by the substrate, through introduction of another surface-active molecule or by adding additional ions. ${ }^{10,20,21}$ Under suitable conditions this behaviour gives rise to selective adsorbtion. ${ }^{21}$

Surface-enhanced Raman spectra are usually obtained at a negative potential (in this paper all potentials mentioned are relative to SCE) in the electrochemical cell. In the region from 0 to $-900 \mathrm{mV}$, the non-Faradaic region, no electrochemistry occurs for the DNA bases. The spectral changes observed as a function of potential ${ }^{17}$ in this region are therefore attributed to subtle changes in the charge distribution of the metal surface-molecule complex and in the potential- 
dependent changes in orientation and concentration. ${ }^{6}$ Where electrochemical processes or photochemistry occur at the metal interface, surface-enhanced Raman scattering may provide information on the sequence of events and the type of molecules formed during the reactions.

In this paper the surface-enhanced Raman spectra of adenine and dAMP adsorbed at a silver electrode maintained at zero or positive potentials are presented. The electrochemical behaviour was investigated by recording the time dependence of complex formation in the case of dAMP. From the time dependence in the spectra two different molecular species are observed to be present.

\section{EXPERIMENTAL}

The buffer solution contains $100 \mathrm{~mm} \mathrm{KCl}$ and $1 \mathrm{~mm}$ $\mathrm{Na}_{2} \mathrm{HPO}_{4}$ and is used for the roughening of the electrode surface. A similar solution, but also containing 1 mM adenine or dAMP, was used for the actual measurements. The $\mathrm{pH}$ was adjusted with either a solution of $\mathrm{NaOH}$ or $\mathrm{HCl}$. The electrochemical SERS cell has been described previously. ${ }^{22}$ The electrode was illuminated with and without laser light during the electrochemical roughening. No difference was found with respect to the observed complex formation.

The roughening was established as follows. At the open loop potential the electrochemical cell was filled with the buffer solution. After connection of the electrodes the potential of the working electrode was increased at a rate of $10 \mathrm{mV} \mathrm{s}^{-1}$. At $\mathrm{ca} .+60 \mathrm{mV}$ the oxidation of silver starts. The potential was increased until it reached $+120 \mathrm{mV}$. The current did not exceed ca. $25 \mathrm{~mA}$. The potential was then reversed. At $c a .+40$ $\mathrm{mV}$ the oxidation current changed to a reduction current. The reduction current increased to $-50 \mathrm{mV}$. Currents larger than $c a .25 \mathrm{~mA}$ were again avoided. When all the $\mathrm{Ag}^{+}$was reduced to silver at $-100 \mathrm{mV}$ the potential was changed to the open loop potential. The electrodes were disconnected and the buffer solution was replaced with the solution containing the compound of interest.

A SPEX 1877 0.6-m triple monochromator was used in conjunction with an EG\&G/PARC optical multichannel analyser system. The time resolution used to obtain the time-dependent changes was $10 \mathrm{~s}$. A final time resolution of $40 \mathrm{~s}$ was used in the spectra presented in Figs 4 and 5. These spectra result from averaging four consecutive scans. A Spectra-Physics Model 2025-03 argon ion laser was used for the excitation of the Raman spectrum. The power at the sample was $1 \mathrm{~mW}$ and the wavelength used was $514.5 \mathrm{~nm}$. The spectra were obtained using backscattering geometry under a microscope. An objective with a ten times magnification produced a spot size of $c a .6 \mu \mathrm{m}$.

\section{RESULTS}

Introduction of the adenine (dAMP) solution after the roughening cycle was performed and subsequent spon- taneous adsorption on the silver working electrode permits the observation of a spectrum of adenine [Fig. 2(d)] or dAMP at open loop potential. This spectrum is in accordance with the spectrum obtained in the nonFaradaic voltage region (Fig. 3 and Refs 4-7). This spectrum completely disappears when the potential is made more positive than $+60 \mathrm{mV}$.

A second excursion in the positive potential region in the presence of adenine causes the production of a strong spectrum after the start of the oxidation of silver at point $\mathrm{A}$ (Fig. 1), at ca. $+70 \mathrm{mV}$. This spectrum is shown in Fig. 2 for pH (a) 11.0 (b) 7.5 and (c) 3.0. The strong ring stretching vibration, which is the most

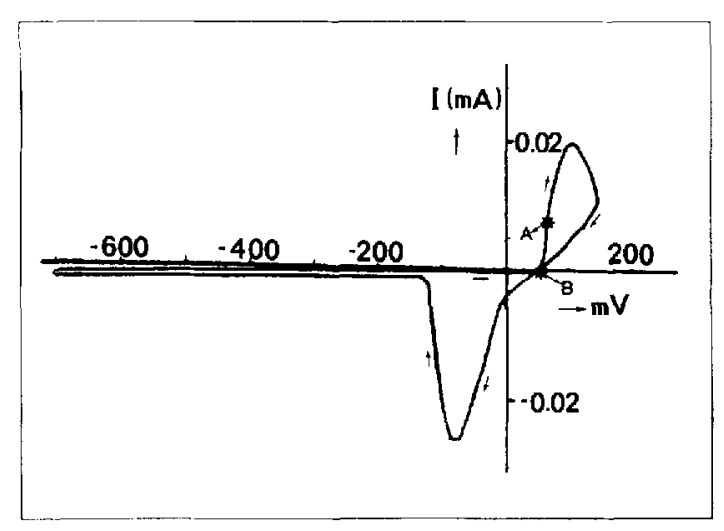

Figure 1. Voltage-current diagram of the oxidation-reduction cycle of a silver electrode in a solution containing $\mathrm{Cl}^{-}$ions. The direction of the loop is indicated with arrows. Positions $A$ and $B$ indicate where the onset of the increase in intensity of the SERS spectrum of the $\mathrm{Ag}^{+}$-adenine complex and that of the $\mathrm{Ag}^{+}-\mathrm{dAMP}$ complex is observed, respectively. A lies in the anodic part of the sweep whereas B occurs where an oxidizing current reverses to a reducing current.

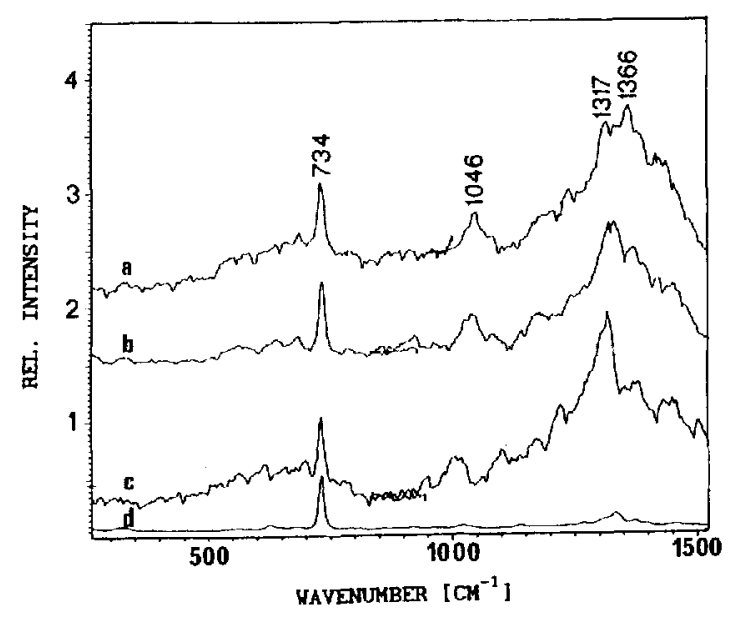

Figure 2. Spectra of adenine at a potential of $+70 \mathrm{mV}$ at various $\mathrm{pH}$ values: (a) 11.0 ; (b) 7.5 ; (c) 3.0. (d) Spectrum obtained after the addition of buffer with adenine at an open loop potential. Spectra (a), (b) and (c) were obtained as two different measurements. The vertical scale of the part of the spectrum from 260 to $900 \mathrm{~cm}^{-1}$ therefore bears no meaningful relationship to the righthand part of the spectrum owing to the time dependence of the signal. Under the conditions for which spectrum (d) was recorded no time dependence is present and the spectra have therefore been joined together. It is proposed that spectra (a)-(c) result from photo-oxidation products of an adenine-silver complex (see text). 


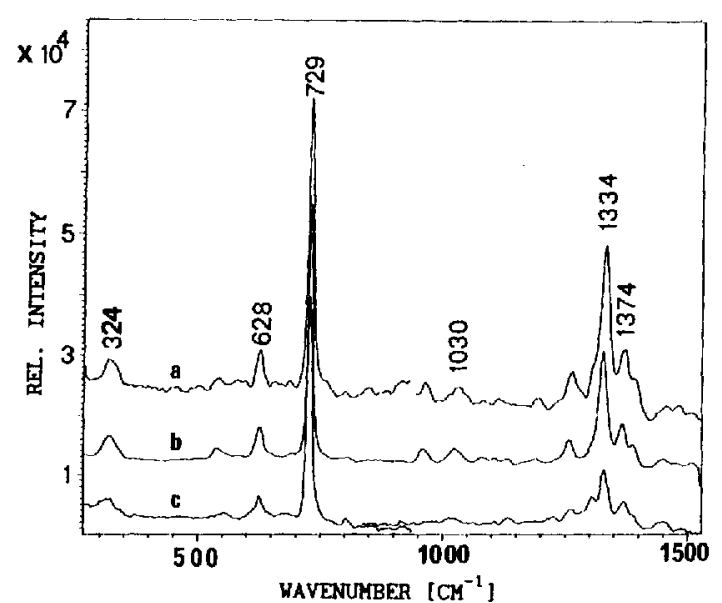

Figure 3. $\mathrm{pH}$ dependence of the SERS spectra of adenine in the non-Faradaic region at a potential of $-0.7 \mathrm{~V}$. $\mathrm{pH}$ : (a) 11.0 ; (b) 7.5; (c) 3.0. The most important distinction between protonated and non-protonated adenine at these potentials is the resolution of the band at $1308 \mathrm{~cm}^{-1}$ at a low pH from the strong band at 1335 $\mathrm{cm}^{-1}$.

prominent band in the SERS spectrum of adenine at potentials in the non-Faradaic region, ${ }^{6,17}$ disappears rapidly while an increase is observed in the intensity of a strong, largely unresolved band in the single-bond stretching region. The occurrence of a broad band around $1350 \mathrm{~cm}^{-1}$ is not $\mathrm{pH}$ dependent. The exact shape is slightly $\mathrm{pH}$ dependent. Most notable is the decrease in the intensity of the band at $1329 \mathrm{~cm}^{-1}$ with increase in $\mathrm{pH}$ (Fig. 2). The $\mathrm{pH}$ dependence at a potential of $-0.7 \mathrm{~V}$ in the non-Faradaic region is illustrated in Fig. 3, where the SERS spectra at $\mathrm{pH} 11.0,7.5$ and 3.0 are shown. Protonation of the adenine ring system causes the band at $1308 \mathrm{~cm}^{-1}$ to be resolved from that

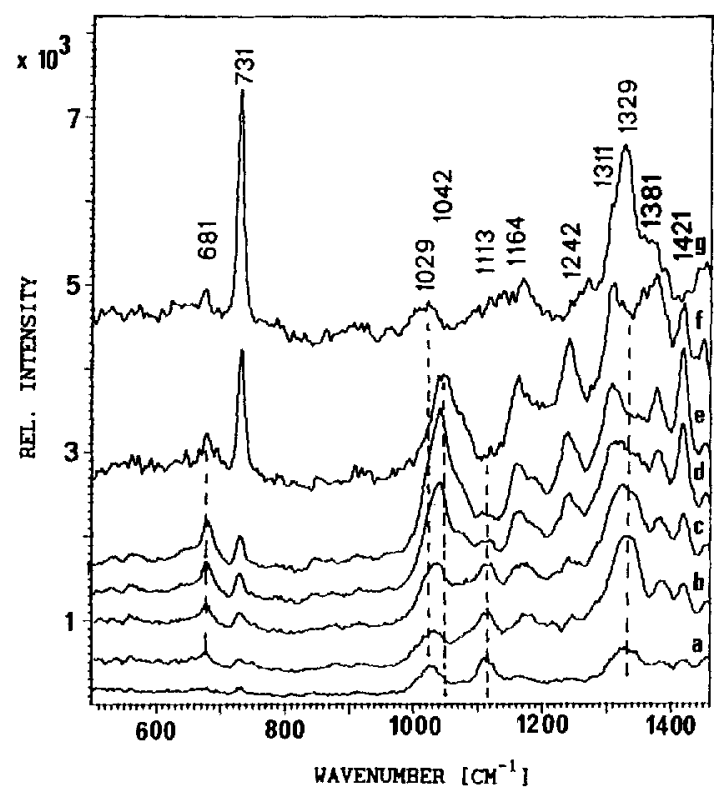

Figure 4. Time dependence of the product formation in the presence of dAMP and $\mathrm{Ag}^{+}$at a pH of 7.5. The spectra were obtained at $0.0 \mathrm{~V}$. (a) Directly after the start of the reduction. Bands are observed at 1030, 1114 and $1331 \mathrm{~cm}^{-1}$. (b)-(f) Spectra obtained with a time difference of $40 \mathrm{~s}$. The initial spectrum is replaced by a spectrum with bands at $681,1042,1163,1242,1310,1380$ and $1421 \mathrm{~cm}^{-1}$. (g) Spectrum obtained after $15 \mathrm{~min}$, showing the recovery of the 'classical' spectrum of dAMP at silver surfaces.

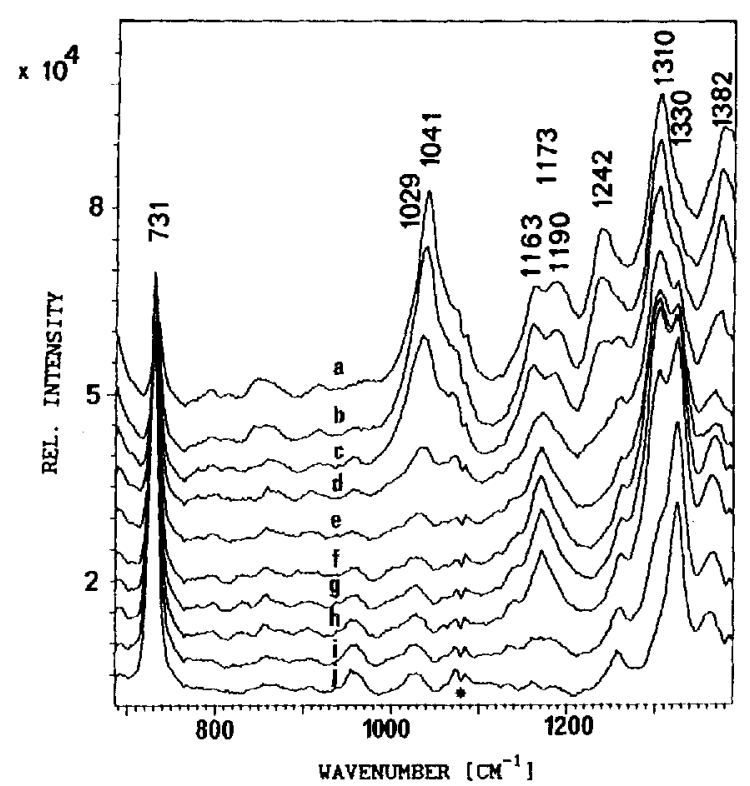

Figure 5. Time dependence of the product formation in the pre sence of dAMP and $\mathrm{Ag}^{+}$at a $\mathrm{pH}$ of 11.0 and at open loop potential. Spectrum (a) is similar to the spectrum in Fig. 4(e). The typical bands at 1042,1163,1242,1310 and $1380 \mathrm{~cm}^{-1}$ are again observed to decrease with time, which increases in steps of $40 \mathrm{~s}$ from (b) to (j). The gradual recovery of the 'classical' SERS spectrum of dAMP is observed. The asterisk indicates an artefact caused by the detector.

at $1334 \mathrm{~cm}^{-1}$ [Fig. 3(c)]. At all potentials the characteristic and strong totally symmetric ring stretching vibration is present. The exact peak position of this band depends on the potential but is $729 \mathrm{~cm}^{-1}$ at $-700 \mathrm{mV}$.

The presence of dAMP during a second oxidationreduction cycle causes the occurrence of a new spectrum at point $\mathbf{B}$ in Fig. $1(\mathrm{ca} .+50 \mathrm{mV})$, where the oxidation current changes to a reduction current. Starting at open loop potential until $c a .+60 \mathrm{mV}$, the totally symmetric ring stretching vibration is observed. At point B an intense spectrum is observed. The time dependence of the formation of a new product can be followed. Figure 4 shows different stages of complex formation. These

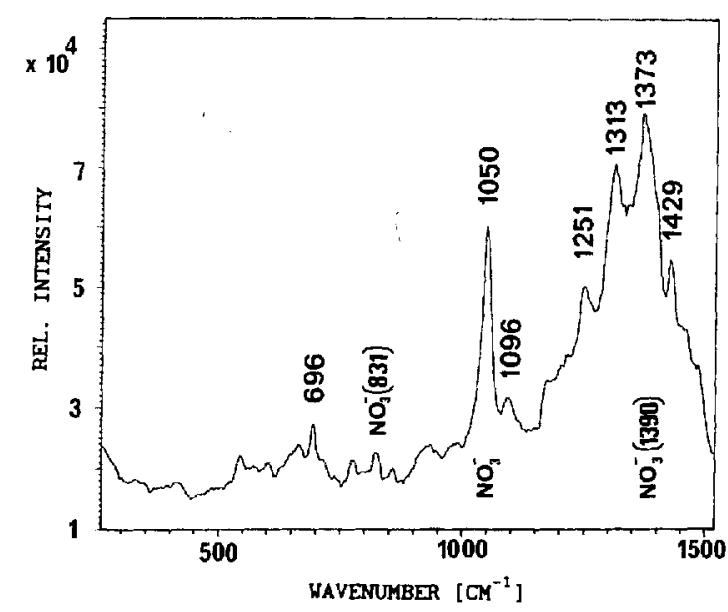

Figure 6. Raman spectrum of a mixture of a solution of silver nitrate with dAMP at a pH of 7.5 (before mixing). Bonds originating from the $\mathrm{NO}_{3}{ }^{-}$group are indicated. The broad band structure between 1200 and $1500 \mathrm{~cm}^{-1}$ and the typical band positions of photochemical products of dAMP are again observed. 
spectra were recorded at a pH of 7.5. Figure 4(a) was recorded directly after the start of the reduction at point B. Weak bands are found at 1030, 1114 and $1331 \mathrm{~cm}^{-1}$. The very weak band at ca. $733 \mathrm{~cm}^{-1}$ is due to a small amount of dAMP adsorbed on the silver surface and its position is in accordance with the position of the symmetric ring stretching mode at this potential. The consecutive scans in Fig. 4 reflect, with a 40 -s time resolution, the development of a new product at the surface. This product is characterized by bands at 681 , $1042,1164,1242,1310,1380$ and $1421 \mathrm{~cm}^{-1}$. None of these band positions coincide with bands observed in the SERS spectrum of dAMP at non-Faradaic potentials (Fig. 3). Hence these bands must be due to a new surface species that is produced when $\mathrm{Ag}^{+}$ions are released from the $\mathrm{AgCl}$ layer to undergo reduction. After ca. $3 \mathrm{~min}$ a band at $731 \mathrm{~cm}^{-1}$ can be observed to increase in intensity [Figs 4(e) and (f)]. A further increase in the intensity of this band is observed in Fig. $4(\mathrm{~g})$ after a longer period of time (typically $10 \mathrm{~min}$ ). This band corresponds to the totally symmetric ring stretching vibration of dAMP, which can also be observed at negative potentials.

In Fig. 5 the results are shown for dAMP at $\mathrm{pH} 11.0$. The characteristic bands of the complex formed at the surface are again present at positions $1042,1163,1242$, 1310 and $1382 \mathrm{~cm}^{-1}$. The spectrum in Fig. 5(a) is similar to that in Fig. 4(e) and Figs $5(\mathrm{a})-(\mathrm{j})$ show the replacement of this product by the 'classical' dAMPsilver complex, characterized by the bands also observed in Fig. 3. The product generated at the surface is only slowly replaced by the dAMP-Ag complex. A residual scattering from the initial product centred around $1300 \mathrm{~cm}^{-1}$ is observed even after hours.

Measurement of the $\mathrm{pH}$ in the electrochemical cell simultaneously with the change in potential reveals a decrease in the $\mathrm{pH}$ as $\mathrm{Ag}^{+}$produced at positive potentials interacts with adenine. A decrease in $\mathrm{pH}$ also occurs in a solution (initially at $\mathrm{pH} 7.5$ ) containing a $1: 1$ ratio of adenine (dAMP) to $\mathrm{Ag}^{+}$. Mixtures of a solution of $10^{-5} \mathrm{M}$ dAMP with solutions containing various amounts of $\mathrm{Ag}^{+}$become turbid at a dAMP to $\mathrm{Ag}^{+}$ratio of 100 . This behaviour indicates a low solubility of the silver-adenine (dAMP) complexes. The Raman spectrum of a solution containing $\mathrm{Ag}^{+}$(and $\mathrm{NO}_{3}{ }^{-}$) and dAMP was obtained. The spectrum presented in Fig. 6 should not be used for quantitative purposes, however, as the sample was not stable in the laser beam. The vibrational bands of the nitrate ion at 831 , 1050 and $1390 \mathrm{~cm}^{-1}$ are observed ${ }^{23}$ and the spectrum also shows that silver-dAMP complexes give rise to an intense spectrum in the $1200-1500 \mathrm{~cm}^{-1}$ region.

\section{DISCUSSION}

Silver ions belong to the class B metals, ${ }^{16}$ which act as Lewis acids. Silver ions preferentially interact with purine or pyrimidine residues in DNA rather than with the charged phosphates. ${ }^{24}$ Several workers have proposed models for $\mathrm{Ag}^{+}$-adenine interactions. ${ }^{15,24,25}$ Some of the models proposed ${ }^{24}$ depend on the ratio, $R$, of the concentration of the silver ion versus the nucleotide. The critical value of $R$ for the formation of a so- called type I complex is $\mathrm{pH}$ dependent. $R$ decreases from 0.20 for $\mathrm{pH} 5.6$ to 0.065 for $\mathrm{pH} 8.0$. It follows that at high $\mathrm{pH}$ very low concentrations of $\mathrm{Ag}^{+}$already lead to the formation of type I complexes.

$R$ varies from 0.2 to 0.5 for a type II complex. Deprotonation occurs in complexes of this type. A silver ion displaces one of the protons participating in the Watson-Crick base-pairing scheme. This may occur both for GC base pairs and for AT base pairs. The N-1 atom of adenine is linked to a silver ion in this structure.

For $R>0.5$ and $\mathrm{pH}<7$ a precipitate is formed. Type III complexes occur in this precipitate. ${ }^{24}$

Recently it was suggested ${ }^{25}$ that polymer chains of adenine- $\mathrm{Ag}^{+}$complexes are formed in solutions with a silver ion to adenine ratio higher than 1.4 . It was proposed that the chain consisted of an alternating adenine-silver complex, with the silver positioned between the N-9 atom of an adenine molecule and the $\mathrm{N}-1$ atom of the next adenine molecule. ${ }^{25}$ The hydrogen atom on the N-9 atom is displaced in this process. The occurrence of $\mathrm{N}-1-\mathrm{Ag}^{+}-\mathrm{N}-1$ complexes and $\mathrm{N}-9-\mathrm{Ag}^{+}-$ $\mathrm{N}-9$ complexes together with the $\mathrm{N}-9-\mathrm{Ag}^{+}-\mathrm{N}-1$ complex, leading to polymer chain branching, could not be ruled out. At higher stoichiometric ratios of silver to adenine the polymer degraded, possibly into dimers. ${ }^{25}$

The experimental results obtained with SERS may be understood when we consider the chemical composition of the dielectric layer and the solution at positive surface potentials. The conditions at the silver working electrode in the electrochemical cell vary markedly with the working electrode voltage. At positive voltages high $\mathrm{Ag}^{+}$concentrations are present in the dielectric layer at the surface where adenine (dAMP) is also present. Adenine may participate in any of the above-mentioned types of complexes. It is assumed that high concentrations of $\mathrm{Ag}^{+}{ }^{+}$-adenine complexes, and possibly the type of polymers proposed by Matsuoka et al., ${ }^{25}$ accumulate at the surface. Deprotonation occurs during this process. All these complexes will, to some extent, be photosensitive as $\mathrm{Ag}^{+}$itself is photoreactive. Therefore, a laser-induced reduction of $\mathrm{Ag}^{+}$is likely to occur. In the region where the laser beam illuminates the surface these complexes are degraded and oxidation products of adenine accumulate. The spectrum of a complex mixture of Ag-adenine complexes and their photooxidation products is proposed to produce the spectra presented in Fig. 2. The sequence of events described above can be summarized by the following reactions:

$$
\mathrm{Ag}^{+}+\text {adsorbate }_{\text {red. }} \rightleftharpoons \mathrm{Ag-adsorbate} \text { deprot. }+\mathrm{H}^{+}
$$

$$
1
$$

$$
\mathrm{Ag} \text {-adsorbate } \text { deprot. }+h v \rightarrow \mathrm{Ag}+\text { adsorbate }_{\mathrm{ax}}
$$

The reactions are a simplification of the actual situation as oxidized silver atoms will also react with $\mathrm{Cl}^{-}$ ions. A mixture of various silver complexes may therefore be present at the surface. Laser-induced effects on the geometry of the surface roughness and possibly the composition of the SERS active surface have been proposed before. $^{26}$

In case of $\mathrm{dAMP}$, only complex formation of $\mathrm{Ag}^{+}$via the N-1 or N-7 nitrogen atom is possible. Ag-dAMP 
complexes will be present in the dielectric layer at the surface, but no large polymer chains will be formed. A limited number of complexes will contribute to the spectra. This leads to the increased resolution in the spectra of dAMP adsorbed on the surface as compared with the spectra of adenine adsorbed on the surface. The initial spectrum, observed in Fig. 4(a) and characterized by broad bands at 1030,1114 and $1331 \mathrm{~cm}^{-1}$, is therefore believed to be due to the deprotonated product of dAMP with $\mathrm{Ag}$ indicated as 1 in the above reactions. The product that is formed in the laser beam at point B (Fig. 1) is characterized by bands at 681 , $1042,1163,1242,1310,1380$ and $1421 \mathrm{~cm}^{-1}$ is indicated as 2 in the above reactions.

In two reports, ${ }^{7,8}$ spectra have been presented that resemble the spectra in Figs 4 and 5 . In the first study, ${ }^{7}$ oxidation-reduction cycles were performed in the presence of dAMP and a SERS spectrum with characteristic Raman bands at 678, 1039, 1161, 1242, 1309, 1382 and $1419 \mathrm{~cm}^{-1}$ was reported at a potential of $+20 \mathrm{mV}$. These positions are very close to the positions reported here for a photo-oxidation product of $\mathrm{Ag}$-dAMP complexes. The spectrum was assigned to dAMP residues adsorbed flat-on to the surface. The potential-dependent change in orientation ${ }^{7}$ from an attachment with N-7 at potentials more negative than $-200 \mathrm{mV}$ to flat-on orientation at positive potentials is not supported by the present observations. In Figs 4 and 5 it can be observed that both at $\mathrm{pH} 7.5$ and 11.0 a slow increase in the 'classical' dAMP spectrum occurs, even at a $0.0 \mathrm{~V}$ potential. No time dependence was reported. ${ }^{7}$

In the other study, the adsorption of DNA to silver colloidal particles was investigated. The SERS spectra showed intense bands from 1100 to $1500 \mathrm{~cm}^{-1}$. No band was present at $729 \mathrm{~cm}^{-1}$. The spectra were reported to be time dependent, reaching stability after $18 \mathrm{~h}$. A number of bands coincided with bands presently ascribed to Ag-dAMP or photo-oxidation products. The SERS spectra of DNA adsorbed on silver sol are more complicated than the spectra in Figs 4 and 5 owing to the presence of guanine, cytosine and thymine. These compounds are also known to react with silver ions. ${ }^{24}$ Special interactions of silver ions with base pairs have also been proposed as well. ${ }^{24.25} \mathrm{Ag}^{+}$ions may be present in preparations of silver sols as a result of an incomplete reaction of these ions with the reducing agent during the production of the sol. The position of the totally symmetric ring stretching mode of adenine
(dAMP) has been shown ${ }^{17}$ to be useful as an in situ probe of the surface potential. A high value of the frequency of this vibration corresponds to a positive surface potential. The frequency was found ${ }^{8}$ to be 735 $\mathrm{cm}^{-1}$, corresponding to a surface potential of $\mathrm{ca} .+100$ $\mathrm{mV}$. This is sufficiently positive for $\mathrm{Ag}^{+}$ions to exist in the sol particle.

The most important characteristics of the spectra obtained at positive potentials of the silver working electrode is the absence of the intense totally symmetric stretching mode of adenine (dAMP) and the presence of intense and broad bands in the single-bond stretching region. After an oxidation cycle these bands are slowly replaced by the bands that can be attributed to adenine (dAMP) adsorbed at the metallic silver surface. This spectrum is well known from previous work ${ }^{2,4-6,11}$ and is also shown in Fig. 2 as a function of pH. The incomplete replacement of the photochemical products by adenine (dAMP) leads to an increase in the intensity in the $1100-1500 \mathrm{~cm}^{-1}$ region, as can be observed in Figs 4 and 5 even after hours.

\section{CONCLUSIONS}

The simultaneous presence of $\mathrm{Ag}^{+}$ions at positive potentials of the silver working electrode in the electrochemical cell leads to the formation of $\mathrm{Ag}^{+}$ adenine(dAMP) complexes. The formation of these complexes is accompanied by a decrease in $\mathrm{pH}$ due to deprotonation. These complexes will be photoreactive owing to the presence of complexed silver atoms. Photo-oxidation products of adsorbate molecules will be produced in the laser beam. The changes in the spectra as a function of time were followed using a multi-channel Raman spectrometer. The results presented in this paper exemplify the usefulness of SERS as a method for detecting and studying reactions at surfaces. A comparison of the spectra presented with spectra previously reported in the literature provides an alternative interpretation for SERS spectra of DNA and derivatives at the surface of silver colloidal particles and at the surface of a silver electrode. It is found that the behaviour at a positive working potential in the electrochemical cell can also be used to explain some results obtained at silver sol surfaces.

\section{REFERENCES}

1. T. M. Cotton, in Spectroscopy of Surfaces. (Advances in Spectroscopy, Vol. 16), edited by R. J. H. Clark and R. E. Hester, Chapt. 3. Wiley, Chichester (1988).

2. I. Nabiev, R. Efremov and K. Sokolov, in Spectroscopy of Biological Molecules: State of the Art, Proceedings of the Third European Conference on the Spectroscopy of Biological Molecules, edited by $A$. Bertoluzza, C. Fagnano and P. Monti, pages 17-20. Societa Editrice Esculapio. (1989).

3. (a) J. I. Gersten and A. Nitzam, 89; (b) M. Kerker, D.-S. Wang, H. Chew, O. Siiman and G. A. Bumm, 109 in Surface Enhanced Raman Scattering; eds.R. K. Chang and F. E. Furtak, Plenum Press, New York, London (1982).

4. K. M. Ervin, E. Koglin, J.-M. Sequaris, P. Valenta and H. W. Nurnberg, J. Electroanal. Chem. 114, 179 (1980).

5. V. Brabec and K. Niki, Biophys. Chem. 23,63 (1985).
6. C. Otto, F. F. M. de Mul, Huizinga and J. Greve, J. Phys. Chem. 92, 1239 (1988).

7. T. Watanabe, $O$. Kawanami, H. Kaoh, K. Honda, Y. Nishimura and M. Tsuboi, Surf. Sci. 158, 341 (1985).

8. K. Kneipp and J. Flemming, J. Mol. Struct. 145, 173 (1986).

9. C. Otto, T. J. J. van den Tweel, F. F. M. de Mul and J. Greve, J. Raman Spectrosc. 17, 289 (1986).

10. S. K. Kim, T. H. Joo, S. W. Suh and M. S. Kim, J. Raman Spectrosc. 17, (1986).

11. J. S. Suh and M. Moskovits, J. Am. Chem. Soc. 108, 4711 (1986).

12. J.-M. Sequaris, E. Koglin, P. Valenta and H. W. Nurnberg, Ber. Bunsenges. Phys. Chem. 85, 512 (1981).

13. E. Koglin, J.-M. Sequaris and P. Valenta, J. Mol. Struct. 60, 421 (1980) 
14. E. Koglin, J.-M. Sequaris, J.-C. Fritz and P. Valenta, J. Mol. Struct. 114, 219 (1984).

15. T. M. Theophanides in Infrared and Raman Spectroscopy of Biological Molecules, edited by T. M. Theophanides, pp. 187-204 and 205-223. Reidel, Dordrecht (1979).

16. S. Ahrland, J. Chatt and N. R. Davies, Q. Rev. Chem. Soc. 12, 265 (1968).

17. C. Otto, G. W. Lucassen and J. Greve, in XIIth International Conference on Raman Spectroscopy, edited by J. R. Durig and J. F. Sullivan, p. 326. Wiley, Chichester (1990).

18. J. A. Creighton, C. G. Blatchford and M. G. Albrecht, J. Chem. Soc., Faraday Trans, // 75, 790 (1979).

19. L. Sander, Internal Report, University of Twente (1989).

20. P. Hildebrandt and M. Stockburger, J. Phys. Chem. 88, 5935 (1984).
21. I. R. Nabiev, K. V. Sokolov and O. N. Voloshin, J. Raman Spectrosc. 21, 333 (1990).

22. C. Otto, A. G. M. van Welie, E. de Jong. F. F. M. de Mul, J. Mud and J. Greve, J. Phys. E 17, 624 (1984).

23. G. Herzberg, Molecular Spectra and Molecular Structure, Part II. Infrared and Raman Spectra of Polyatomic Molecules, van Nostrand. p. 178. (1968).

24. R. H. Jensen and N. Davidson, Biopolymers 4, 17 (1966).

25. Y. Matsuoka, B. Norden and T. Kurucsev, J. Cryst. Spectrosc. Res. 15, 545 (1985)

26. M. R. Mahoney and R. P. Cooney, J. Phys. Chem. 87, 4589 (1983). 